\title{
An Inclusive Stance With a Critical Edge
}

\author{
Katrina McFerran ${ }^{1}$ * \\ 1 University of Melbourne \\ *k.mcferran@unimelb.edu.au
}

Published: 1 July 2017

VOICES is a journal that "invites dialogue and discussion about music, health, and social change." Many of the contributors to the journal are professional music therapists whose university training has been focused entirely on how the affordances of music can, and have, been used to promote a range of opportunities for people with diverse life experiences. Each university training program teaches different theoretical emphases, so whilst there is broad agreement about the international practice of music therapy, there are specific, local differences. Some of these are cultural and others are personal. Further, each music therapist then has the freedom to decide what their particular approach will be in the institution, community organization, or private practice setting where they work. This is usually in response to the perceived needs of the people who participate in music therapy but is naturally influenced by a range of factors.

The editorial team at VOICES has chosen to position our journal so that it is open to contributions from around the world that represent the full range of positions. We "value inclusiveness and socio-cultural awareness and have increasingly nurtured a critical edge that refines the focus on cultural issues and social justice." We encourage authors to position themselves so that their own particular stance is made clear, but we celebrate the full spectrum of manuscripts that emerge from diverse practices. To that end, we have a range of sections of the journal, including Research, Case Stories, Interviews, Columns and Essays, Position Papers, Reports, as well as Book Reviews and Book Essays.

We have increasingly tailored our reviewing processes to match these values with high levels of dialogue and transparency. In this edition, we are including the names of reviewers as well as the names of our abstract translators from around the globe. This affords the reader potential understandings about how some of the diversity in articles results from the different cultural and theoretical positions of reviewers, as well as the authors. It also acknowledges the tremendously important voluntary contributions made by members of our editorial board and translation team.

The result of maintaining an inclusive stance with a critical edge and a commitment to making knowledge available to people around the globe via open access demands ongoing reflexivity. To this end, the editorial team is constantly engaged in discussion and reflection on how to best represent the values of our journal in the manuscripts we publish, and this is actively incorporated into our reviewing process. Although all the articles in the current edition represent music therapists' perspectives, they demonstrate a range of positions and varying degrees of emphasis on socio-cultural awareness. A number of articles in this edition focus on infants and young children. However the aspects of practice that are described with this age group ways are diverse, and the emphasis on research, practice, and theory vary considerably. Kirsi Maaria Tuomi, Ala-Ruona Esa, and Amelia Oldfield provide an overview of 22 years of literature on music therapy and early childhood and highlight an increasing emphasis on fam- 
ily involvement and positive factors. This is certainly illustrated in this edition, with a range of authors emphasizing the value of working with families using vignettes as well as qualitative and quantitative case studies. This is evidenced in Mark Ettenberger's reflections on music therapy practice during end-of life care in the NICU in Colombia that highlights ritual, culture, and memory making with parents and infants. It is also emphasized by nearby colleagues in Brazil, but these authors choose a very different approach. Although focusing on the subjective notion of empowerment, Ambra Palazzi, Rita Meschini, and Cesar Augusto Piccinini have used quantitative methods to examine a music therapy intervention with a mother-preterm infant dyad, also in the NICU. Jess O' Donoghue also emphasises effectiveness but uses qualitative data generated through observations to examine parent-child interactions for families with children who have Down syndrome. Jessica June Nagel and Michael Joseph Silverman also use qualitative data to explore the development of parenting skills, but this time interviewing music therapists to gather their perspectives from work with families experiencing poverty in the USA. One further perspective is offered on music therapy in the context of families, this time exploring the affordances of music to support the recovery of a survivor of childhood abuse. Georgina Lewis uses auto-ethnographic analysis to reflect on how music serves different functions during four stages of recovery.

This edition is not only focused on childhood experiences however, with two manuscripts that explicitly argue for the integration of different theoretical frameworks into music therapy practice. Rosemyriam Cunha focuses on the framework of musicking from the discipline of musicology, emphasizing collective music making with groups to promote positive change. Niels Hannibal and Melody Schwantes provide a rationale for the integration of mentalization into music therapy practice, based on extensive experience working with people who have borderline personality disorder.

This edition of VOICES certainly addresses our desire for inclusivity and offers a range of theoretical perspectives and research approaches from around the globe. We hope this diversity affords you, the reader, an opportunity to engage with ideas that naturally resonate with your own stance. We also hope you may find something outside of that which you knew already. Studies of human nature suggest that it is much more difficult for us to tolerate and integrate ideas that do not match our own, but we aim to provide opportunities which you may take or leave. This is the great advantage of an open-access forum that does not require you to financially commit before reading, and therefore allows you to access knowledge from wherever you are, at any time, when and if you choose. Happy reading. 